\title{
Mujer Indígena y Cuidados Durante el Embarazo y Puerperio
}

Por:

Magister Marta A. Acosta C. Candidata Doctoral. Programa de Doctorado en Enfermería con Énfasis Salud Internacional. Profesora Agregada de Administración en los Servicios de Salud en el Centro Regional Universitario de Veraguas, (CRUV). Universidad de Panamá. E mail: macosta9 @ hotmail.com

Diamantina Cleghorn Spencer. MPH; PhD; Estudios Post Doctoral en Salud Pública Global.

Profesora Titular de Salud Pública. Departamento de Salud Pública. Facultad de Enfermería de Enfermería. Universidad de Panamá. E mail: diacleghorn@ hotmail.com

\section{Resumen}

En los albores de este siglo XXI, con un indiscutible escenario de grandes avances de la ciencia y la tecnología, se presenta la realidad con la que la población de pueblos originarios resiste, con urgentes modificaciones y efectos resilientes para dar sentido a la vida que le corresponde aceptar, sin sucumbir a su incierto destino.

Los pueblos originarios de nuestra América, al igual que en Panamá, con los escasos recursos que logra conseguir para su supervivencia, los Ngäbe-Buglé y otros pueblos indígenas, efectúan ingentes esfuerzos para la supervivencia. De manera que ser pobre, ser indígena y mujer, en un área rural, representa un gran reto para desafiar a la vida y el universo.

Objetivo. Presentar resultados en relación con investigaciones efectuadas en torno a la temática de la mujer indígena y su salud durante el embarazo y el puerperio.

Metodología. Revisión de artículos científicos, de investigaciones realizadas en América, en relación con la mujer indígena y los cuidados durante el embarazo y el puerperio, en las principales bases de datos disponibles en ciencias de la salud y de enfermería.

Resultados. Las mujeres acceden a los servicios de salud con las características de doble desventajas: ser mujer y ser indígena. El significado que le asigna la mujer a su cuidado durante el embarazo y el puerperio es diverso y está influido por el componente cultural, con prácticas y creencias.

Conclusiones. La salud de la mujer indígena debe considerarse como prioridad en la prestación de los servicios de salud. El Objetivo 5 de Desarrollo Sostenible enfoca que se debe "Lograr la igualdad entre los géneros y empoderar a todas las mujeres y las niñas".

Palabras clave: Salud de la Mujer, Servicios de Salud, Embarazo, Periodo Posparto. 


\title{
Indigenous Woman and Care During Pregnancy and Postpartum
}

\begin{abstract}
At the dawn of this 21 st century, with an undeniable scenario of great advances in science and technology, we present the reality with which the population of indigenous peoples resists, with urgent modifications and resilient effects to give meaning to the life that corresponds to accept, without succumbing to its uncertain destiny.

The original peoples of our America, as in Panama, with the scarce resources that the Ngäbe-Buglé and other indigenous peoples manage to obtain for their survival, make enormous efforts for their survival. So being poor, being indigenous and woman, in a rural area, represents a great challenge to challenge life and the universe.

Objective. Present results in relation to research conducted on the topic of indigenous women and their health during pregnancy and the puerperium.

Methodology. Review of scientific articles, research conducted in America, in relation to indigenous women and care during pregnancy and puerperium, in the main databases available in health sciences and nursing.

Results. Women have access to health services with the characteristics of double disadvantages: being a woman and being indigenous. The meaning that the woman assigns to her care during pregnancy and the puerperium is diverse and is influenced by the cultural component, with practices and beliefs.

Conclusions. The health of indigenous women should be considered as a priority in the provision of health services. The Sustainable Development Goal focuses on "Achieving gender equality and empowering all women and girls."
\end{abstract}

Key words: Women's Health, Health Services, Pregnancy, Postpartum Period.

\section{Mulher Indígena e Cuidados Durante a Gravidez e Puerperio}

\section{Resumo:}

No início deste século 21, com um cenário inegável de grandes avanços em ciência e tecnologia, apresentamos a realidade com a qual a população de povos indígenas resiste, com modificações urgentes e efeitos resilientes para dar sentido à vida que corresponde aceitar, sem sucumbir ao seu destino incerto. 
Os povos nativos de nossa América, como no Panamá os Ngäbe-Buglé e outros povos indígenas, com os escassos recursos que conseguem obter fazem enormes esforços para sua sobrevivência. Portanto, ser pobre, ser indígena e mulher, em uma área rural, representa um grande desafio para desafiar a vida e o universo.

Objetivo: Apresentar resultados em relação à pesquisa realizada sobre o tema das mulheres indígenas e sua saúde durante a gravidez e o puerpério.

Metodologia: Revisão de artigos científicos, de pesquisas realizadas na América, em relação às mulheres indígenas e cuidados durante a gravidez e puerpério, nas principais bases de dados disponíveis em ciências da saúde e enfermagem.

Resultados: As mulheres têm acesso a serviços de saúde com características de dupla desvantagem: ser mulher e ser indígena. O significado que a mulher atribui a seus cuidados durante a gravidez e o puerpério é diversificado e é influenciado pelo componente cultural, com práticas e crenças.

Conclusões: A saúde das mulheres indígenas deve ser considerada como uma prioridade na prestação de serviços de saúde. O Objetivo 5 do Desenvolvimento Sustentável enfoca "alcançar a igualdade de gênero e capacitar todas as mulheres e meninas".

Palavras Chaves: Saúde da Mulher, Serviços de Sáude, Gravidez, Período Pós- Parto. Introducción

En esta nueva era de la información y comunicación, en la cual parece que todas las necesidades básicas han sido resueltas para la humanidad.

Sin embargo, hoy en día se encuentra pendiente el reto de la solución a problemas básicos en nuestra humanidad, en el grupo de los pueblos originarios de nuestra América, al igual que en Panamá, con los escasos recursos que logra conseguir, para su supervivencia, éstos efectúan ingentes esfuerzos para la supervivencia.

De manera que, ser pobre, ser indígena y mujer, viviendo en un área rural, representa un gran reto para el desafío a la vida y al Universo. De acuerdo a las ideas anteriores, la situación de la mujer indígena se enuncia en los Objetivos de Desarrollo Sostenibles, los cuales fueron aprobados por 193 Estados Miembros que la suscribieron en la Agenda 2030, en septiembre de 2015, por la Asamblea General de las Naciones Unidas.

De manera que el Objetivo 5, se enfoca en "Lograr la igualdad entre los géneros y empoderar a todas las mujeres y las niñas". Recuperado de:

http://www.un.org/sustainabledevelopment/es/gender-equality/

Desde la perspectiva del estudio titulado Creencias religiosas y prácticas médicas frente al cuerpo de las mujeres indígenas del norte argentino, realizado por Vásquez Laba, V. P. et. al. (2015), las mujeres acceden a los servicios de salud con las características de doble desventajas: ser mujer y ser indígena. 
Por su parte, Noreña Herrera, C.; et. al. (2015), en su estudio Inequidad en la utilización de servicios de salud reproductiva en Colombia en mujeres indígenas y afrodescendientes, refiere que las mujeres indígenas tienen "...menores probabilidades de un número adecuado de controles prenatales $(\mathrm{OR}=0,61) \ldots$...

En cuanto a la atención institucional del parto: indígenas $(\mathrm{OR}=0,33)$. En relación a los controles posparto. Indígenas $(0,80)$. Por lo tanto, existen inequidades en la utilización de los servicios de salud reproductiva en las mujeres de grupos étnicos de Colombia.

De igual manera, Leyva Flores, R.; et. al (2013), se refieren en su estudio a la Inequidad persistente en salud y acceso a los servicios para los pueblos indígenas de México, 2006-2012, manifestando que el 60\% del total de la población indígena de México, se ubica en el nivel socioeconómico más bajo.

En cuanto a la atención hospitalaria del parto en indígenas aumentó de 63.8 a $76.4 \%$ en población, y continúa la diferencia con no indígenas de $93.9 \%$.

Por lo tanto, no se observan cambios en la atención de salud (de 7.1 a 6.4\%), a pesar de que el Seguro Popular amplió su cobertura de 14 a $61.9 \%$ en población indígena en comparación con la no indígena (de 10 a 35.7\%).

Por otro lado, Prieto Bocanegra, B.; Ruiz Cárdenas, C. H. (2013), en su estudio sobre Significado durante el puerperio: a partir de prácticas y creencias religiosas, señala que el significado que le asigna la mujer a su cuidado durante el puerperio es diverso y está influido por el componente cultural, debido a que las prácticas y creencias surgen a partir de esta dimensión; al igual que los factores socioculturales determinan este cuidado en relación a los valores, creencias y estilos de vida; asimismo los factores sociales y de parentesco. En una menor proporción influyen en el cuidado los factores religiosos, políticos y económicos. De igual manera, la lactancia requiere del apoyo de la familia y del profesional de enfermería, por las diversas dificultades que se presentan en los primeros días de lactancia.

Barrionuevo Rosas, L.; et. al. (2013), señala en su estudio ¿Cómo afecta el tipo de seguro de salud a la realización del Papanicolau en Perú?, que el 62,7\% de las mujeres sexualmente activas se habían realizado algún PAP en los últimos 5 años.

Al igual que, las mujeres que tenían la participación más baja eran las analfabetas o con educación primaria, de nivel socioeconómico bajo, con antecedente de lengua indígena y que vivían en las zonas rurales (siendo esta brecha mayor cuando además no tenían seguro de salud, llegando a ser hasta la tercera parte en relación con los grupos sociales más favorecidos.

Por lo tanto, las mujeres sin seguro suponen una barrera para el acceso a cribado de cáncer de cérvix en Perú.

Otro aspecto a considerar en los grupos de pueblos originarios, de acuerdo con Cabada Ramos, E., et. al. (2015), en su estudio referente a la Prevalencia de los síntomas de depresión posparto en población urbana, rural e indígena, se refiere a que la depresión 
posparto debe ser considerada con base en variables biopsicosociales y su identificación tempranamente.

De igual forma no hay diferencias estadísticamente significativas entre los tres grupos: rural, urbana e indígena. Los resultados identificaron que presentan depresión posparto, en este estudio, un $16 \%$ correspondió a población urbana, un $26 \%$ a la población rural y $27 \%$ a la población indígena.

Se debe considerar la participación de la familia en el bienestar de la gestante y sus cuidados propios, alimentación y actividades, ofrecidos por la familia, que inciden en la protección de la vida de la gestante y del bebé, como lo enuncia, Oviedo. M. P.; et. al. (2013), en su estudio titulado Comunidades Emberá y Wounaan del departamento de Chocó: prácticas de cuidado durante el proceso de gestación.

Otro aspecto a considerar, corresponde a la mediación de las diferencias culturales entre los sistemas médicos y tradicionales, por lo que es necesario la comprensión de la preservación de los cuidados culturales de la familia Nasa y el reconocimiento de la medicina facultativa; lo cual es demostrado en el estudio sobre Interculturalidad y percepciones en salud materno-perinatal, Toribio Cauca 2008-2009, efectuado por Muñoz Bravo, S. F.; et. al. (2012).

Metodología: Se realiza búsquedas en las principales bases de datos disponibles en Ciencias de la Salud y de Enfermería, al igual que The Cochrane Library, Medline, Lilacs y CUIDEN, Latindex, Scielo, Redalyc, Dialnet, EBSCO, NCBI, Fisterra, PubMed.

Se utilizó los descriptores de: mujer indígena, salud indígena, pueblos originarios y salud, salud de la mujer, salud sexual y reproductiva, cuidados, embarazo y puerperio. Se localizó fuentes bibliográficas, de los cuales en una primera selección se escogió los documentos de estudios tanto de tipo cualitativo, como cuantitativo, o con diseños mixtos, apoyados en revisiones bibliográficas de las fuentes descritas.

\section{Resultados.}

Es necesario incidir en los cambios comportamentales con intervenciones educativas en los pueblos originarios hacia las prácticas cultuales de la salud, incluyendo los saberes de la medicina facultativa. Saber integrar los dos saberes, tanto de los pueblos originarios, como de los facultativos de las ciencias de la salud.

Las mujeres que acceden a los servicios de salud, cuentan con doble dificultad y desventaja, establecido en dos factores adversos, ser mujer y ser indígena, debido a los prejuicios étnicos raciales, que han sido motivadas por las creencias religiosas de las mujeres.

De aquí que, en las épocas cruciales de la salud reproductiva como el embarazo, no mantienen un adecuado número de controles prenatales, con riesgo de su salud durante el embarazo, y la de su bebé en gestación. 
De igual manera, el acceso a los servicios de salud, está influido por la tenencia o no de seguro de salud, y aun manteniendo un Seguro Popular, el cual debiera ser garantía de acceso a los servicios de salud, no asegura la atención. Por lo tanto, las mujeres que presentan mayores dificultades, las que son analfabetas o con educación primaria, nivel socioeconómico bajo, antecedente de lengua indígena y que viven en zonas rurales; siendo éstas las que no acuden a sus controles de toma de citología cervical (cribado de cáncer de cérvix).

De la misma manera, las mujeres indígenas son las que no son cubiertas por atención hospitalaria del parto en su totalidad. A pesar de esta situación, las mujeres reclaman su espacio y solicitan que sus derechos sean escuchados, atendidos y resueltos.

La mayoría de los sistemas de salud de hoy en día, no reconocen la importancia de las parteras y no se toman en consideración los aspectos socioculturales de cada comunidad y país. De manera que las mujeres indígenas, en circunstancias actuales y de acuerdo a las situaciones de vida, manifiestan resistencia por ser atendidas por médicos.

Más bien, algunas etnias prefieren realizar prácticas de cuidados según sus creencias culturales, y es practicada por las mujeres. Prefieren realizar prácticas de cuidados que incidan en la salud durante el puerperio y cuidado del hijo, a través del secreto de la medicina tradicional.

Debido a las dificultades que las mujeres indígenas presentan y confrontan para acceder a los servicios de salud oportunamente, en algunos estudios se refleja la necesidad de atención a la salud, en que se deben considerar las diferencias culturales y étnicas.

Es meritorio mencionar que la mayoría de los sistemas de salud de hoy en día, no reconocen la importancia de las parteras y no se toman en consideración los aspectos socioculturales de cada comunidad y país.

En general, debe considerarse la salud de la mujer, como la primera prioridad a nivel mundial, por ser la dadora de vida, por lo que es preciso mencionar los aspectos presentados en los Objetivos de Desarrollo Sostenibles, aprobados en la Agenda 2030, en septiembre de 2015 por la Asamblea General de las Naciones Unidas, que involucra a la mujer indígena en el Objetivo 5.

\section{Conclusiones.}

La salud de la mujer, debe representar la preeminencia de reconocimiento a nivel mundial, por ser la facilitadora de toda vida humana, por lo que es preciso enfatizar en los Objetivos de Desarrollo Sostenibles, aprobados en la Agenda 2030, en septiembre de 2015 por la Asamblea General de las Naciones Unidas, con la representación de 193 Estados Miembros que la firmaron con el compromiso de cumplimiento cabal.

Entre los 17 Objetivos de Desarrollo Sostenible establecidos el Objetivo 5 se refiere la Igualdad de Género, es meritorio mencionar aspectos relacionados a la mujer indígena, a pesar de que no se efectúa énfasis específicamente en las etnias originarias. 
Lograr “...la igualdad entre los géneros...” y “...empoderar a las mujeres y las niñas..."

Programa de las Naciones Unidas para el Desarrollo. Entre las metas, se destacan las que a continuación se describen:

- Poner fin todas las formas de discriminación contra todas las mujeres y las niñas en todo el mundo.

Sin embargo, Tubin, V. (2015), manifiesta que "la denigración de las mujeres es parte del sistema patriarcal y racial, con prácticas particulares, que opera con impunidad, legitimidad..." de manera que son formas de violencia que..." "... atentan contra la dignidad de mayas, garífunas y xinkas", a las que, continúa refiriendo..." "se les niega el derecho a ser parte de su territorio, cosmovisión y visión política...".

- Garantizar el acceso universal a la salud sexual y reproductiva y los derechos reproductivos, de conformidad con el Programa de Acción de la Conferencia Internacional sobre la Población y el Desarrollo, la Plataforma de Acción de Beijing y los documentos finales de sus conferencias de examen.

Emprender reformas que otorguen a las mujeres el derecho a los recursos económicos en condiciones de igualdad, así como el acceso a la propiedad y al control de las tierras y otros bienes, los servicios financieros, la herencia y los recursos naturales, de conformidad con las leyes nacionales.

De acuerdo a estas Metas, señaladas en el Objetivo 5 de Desarrollo Sostenible, es preciso, dirigir estrategias que induzcan a la promoción y prevención de la salud sexual y reproductiva de nuestras mujeres, principalmente, las que pertenecen a las diversas etnias culturales de Panamá, Centroamérica y el Mundo.

\section{Referencias:}

Barrionuevo Rosas, L., Palencia, L. y Borrell, C. (2013). ¿Cómo Afecta el Tipo de Seguro de Salud a la Realización del Papanicolau en Perú? Rev. Panamá Salud Pública 34(6), 393-400. Recuperado de: http://www.scielosp.org/pdf/rpsp/v34n6/v34n6a04.pdf

Leyva, R., Infante, C., Gutiérrez, J., y Quintino, F. (2013). Inequidad Persistente en Salud y Acceso a los Servicios para los Pueblos Indígenas de México, 2006-2012. Salud Pública Mex. 55(2), 23-28. Recuperado de: http://www.scielo.org.mx/pdf/spm/v55s2/v55s2a8.pdf

Noreña, C., Leyva, R., Palacio, L. y Duarte, M. (2015). Inequidad en la Utilización de Servicios de Salud Reproductiva en Colombia en Mujeres Indígenas y Afrodescendientes. Cad. Saúde Pública, 31(12).

Recuperado de: http://www.scielo.br/scielo.php?script=sci_arttext\&pid=S0102-

$311 X 2015001202635 \& \operatorname{lng}=$ es\&nrm=iso\&tlng=en

Organización de las Naciones Unidas (2016). Objetivos de Desarrollo Sostenible. Objetivo 5. Recuperado de: http://www.un.org/sustainabledevelopment/es/gender-equality/

Prieto, B. y Ruiz, H. (2013). Significados Durante el Puerperio: A Partir de Prácticas y Creencias Culturales. Chía, 13(1), 7-16. Recuperado de: http://aquichan.unisabana.edu.co/index.php/aquichan/article/view/2763/pdf_1 
Cabada Ramos, E., et. al. (2015). Prevalencia de los Síntomas de Depresión Posparto en Población Urbana, Rural e Indígena. Revista de Especialidades Médico-Quirúrgicas, (20)1, 18-23. Recuperado de: http://www.redalyc.org/articulo.oa?id=47345916004

Oviedo C, M. P., et. al. (2013). Comunidades Emberá y Wounaan del Departamento de Chocó: Prácticas de Cuidado Durante el Proceso de Gestación. Rev. Fac. Nac. Salud Pública, 32(2), 17-25. Recuperado de: http://www.scielo.org.co/pdf/rfnsp/v32n2/v32n2a03.pdf

Muñoz, S., Castro, E., Castro, Z., Chávez, N. y Ortega, D. (2012). Interculturalidad y Percepciones en Salud Materno Perinatal, Toribio Cauca 2008-2009. Rev. Univ. Ind. Santander. Salud, 44. Recuperado de: http://www.scielo.org.co/scielo.php?script=sci_arttext\&pid=S0121-

08072012000100005\&lng=es\&nrm=iso\&tlng=es

Tubin, V. (2015). Ser Mujer y Ser Maya. Recuperado de: https://www.plazapublica.com.gt/content/sermujer-y-ser-maya

Vásquez, V., Rugna, C. y Perazzolo, R. (2014). Creencias Religiosas y Prácticas Médicas Frente al Cuerpo de las Mujeres Indígenas del Norte Argentino. Revista Sociedad y Religión, 25(43), 47-69. Recuperado de: http://www.scielo.org.ar/pdf/syr/v25n43/v25n43a03.pdf

Recibido: 06 de Septiembre 2017

Aprobado: 22 de Noviembre 2017 